\title{
Protocol
}

\section{Protocol of sterile personal protective equipment for surgical personnel against SARS-CoV-2 during the COVID-19 pandemic}

\author{
Osvaldo I. Guevara Valmana*, Maria M. Cavazos Quero, Elias Gil Loaeza, \\ Jesus C. Lopez Sandoval, Jose A. Pereyra Molina, Mario A. Rebolledo Ceballos, \\ Chantal Gonzalez Chavez, Omar D. Gonzalez Gachuz
}

Department of Surgery Postgraduate Division, UNAM, IMSS Mexico City, Mexico

Received: 03 November 2020

Revised: 17 November 2020

Accepted: 18 November 2020

\section{*Correspondence:}

Dr. Osvaldo I. Guevara Valmana,

E-mail: drosvaldoguevara@hotmail.com

Copyright: ( ) the author(s), publisher and licensee Medip Academy. This is an open-access article distributed under the terms of the Creative Commons Attribution Non-Commercial License, which permits unrestricted non-commercial use, distribution, and reproduction in any medium, provided the original work is properly cited.

\begin{abstract}
Background: COVID-19 represents the major pandemic seen the last years generating morbidity and mortality around the world. It is well known the propagation of the virus occurs by air mostly, so it is needed a barrier when the medic personal is treating suspect or confirm patients. Personal protective equipment represents a barrier between the health personnel and the patient during the COVID-19 pandemic. The surgical team during a COVID-19 confirmed o suspicious case procedure requires using PPE to be protected and keep the sterility for the patient safety.

Methods: A team of surgeons from a 100\% COVID-19 hospital of the Mexican institute of social security developed an inner protocol of safe use PPE maintaining sterility for the surgery.

Conclusions: The protocol described provides safety to surgical team and the patient minimizing risk of surgical infections.
\end{abstract}

Keywords: COVID-19, Personal protective equipment, Surgery

\section{INTRODUCTION}

The ongoing outbreak of coronavirus disease 2019 (COVID-19) caused by severe acute respiratory syndrome coronavirus-2 (SARS-CoV-2) in December has been declared a pandemic by the world health organization (WHO) and has imposed a large burden on global medical systems. ${ }^{1}$ This publication is the result of the incessant growth that COVID-19 pandemic has had worldwide, with an exponential rise in deaths and infections around the globe, generating the need for action protocols for health personnel. ${ }^{2}$

Although the mainly indication worldwide accepted is the delay of the elective surgery, patients with potentially fatal pathologies are in need for emergency surgery (appendicitis, biliary surgery, etc.). ${ }^{3}$ Personal protective equipment (PPE) is a physical barrier between healthcare personnel and the infected patient. Different agencies in charge of health regulation have issued various guidelines on the placement and removal of PPE; these protocols have been used for contact with the patient in an environment that does not require sterility. To date, there is not enough evidence on the use of PPE in a context of sterility in a surgical setting. Currently various organizations such as the American college of surgeons, the American association of gastrointestinal and endoscopic surgeons as well as the Mexican association of surgery have elaborated guidelines on surgery in COVID-19 patients in order to limit exposure and execute procedures safely, however, it will eventually be necessary to have a protocol for possible surgeries in this 
patients, in order to follow widely accepted measures, with the proper adaptations for every region in particular. $^{4}$

The purpose of this publication is to present a sterile PPE donning and doffing protocol, with the equipment available in our region, to perform safe surgeries both for the patients and the surgical team.

\section{METHODS}

The surgical dressing protocol was elaborated by a team of three surgeons and five residents adapting the joint protocol of NETEC, WHO and CDC as well as a recommendation by the ACS, taking practical guidelines from all of them, integrating an appropriate one to local needs and resources. ${ }^{4,5}$ Once the protocol was elaborated, initially was tested in a simulation within the operating rooms of the hospital, and evaluated by multiple surgeons with a checklist of its operability and efficacy. Special emphasis should be done with training for the residents guided by the surgeons of the department as part of the surgical curriculum. ${ }^{6}$

\section{Donning}

The circulating nurse will act as dofficer during the process. ${ }^{7}$ To enter the operating room, personnel must use a disposable surgical uniform or a clean scrub for this procedure, as well as shoes of exclusively surgical use. First the staff must leave all their personal belongings outside the surgical area, in case of having long hair, braid it and collected with a bow. Second put on the surgical cap, where all the hair is inserted, double surgical boots and surgical mask must be worn in the transfer to enter the operating room (OR) area. Outside the OR, which must remain closed, perform hand hygiene. Remove the surgical facemask, place N95 respirator and perform the sealing test. Put on a disposable surgical cap and eye protection which can be choose between different options: Face shield mask over the N95 respirator, previously sterilized reusable sterile plastic lenses or reusable sterile plastic goggles. The dofficer must check that the equipment is well placed and in good condition. Hand hygiene should be carried out with the surgical solution available in the hospital. Perform surgical hand scrub with antiseptic solution available in the hospital according the institutional scrubbing procedure. Dofficer must open the OR door. First pair of surgical gloves must be worn with sterile technique. The instrumentalist helps to place surgical gown and circulating nurse will knot it (circulating with non-sterile PPE). Finally place second pair of sterile gloves. The surgeon must approach to the patient at last moment before the surgery to avoid unnecessary exposition to aerosolization from the patient.

In case of contamination, it is possible to change external gloves with help of the surgical nurse. In case of compromise of another part of the PPE, it must be removed under the doffing process and placed again. N95 respirator should be removed only when leaving the OR (Figure 1).

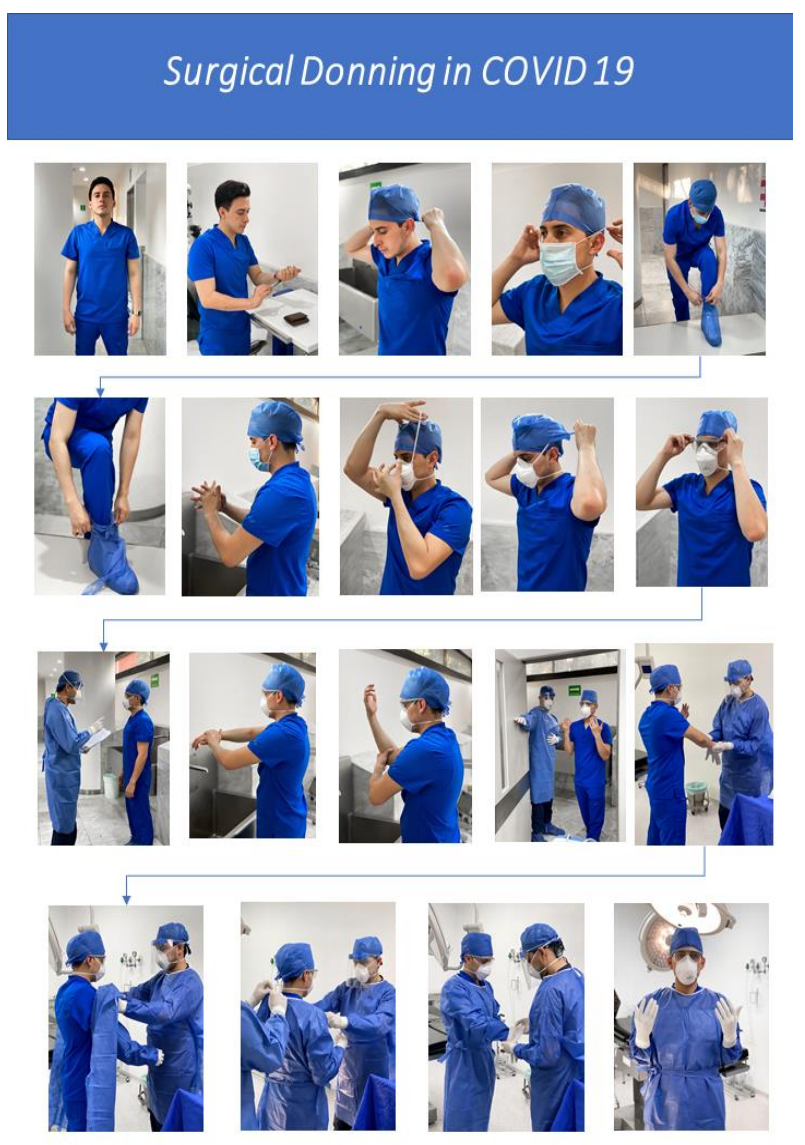

Figure 1: Surgical donning in COVID-19.

\section{Surgical doffing protocol}

It is necessary that circulating nurse in the OR act as dofficer and have the checklist, which must be read and reviewed out loud. First the visible detritus of the upper gloves used in the surgery should be cleaned with wet gauzes. Hand hygiene should be performed with hand sanitizer solution on the second gloves. Second pair of surgical boots should be removed. Hand hygiene on the second gloves should be performed. Disposable gown and second gloves should be removed, unrolling the gloves simultaneously with the gown, together they must be rolled up and placed in their respective bag of biohazardous waste (BHW). Hand hygiene on the first gloves should be performed. The eye protection should be removed and placed in a chlorinated solution if it is reusable, if not, place it in a bag of BHW. Care must be taken to avoid touching the front of the mask or the skin, if it is necessary request help from the dofficer for removal. Hand hygiene on the first gloves should be performed. Disposable external surgical cap should be removed and placed it in BHW bag. Hand hygiene on the first gloves should be performed. First gloves should be removed and place them in BHW bag carefully. Dofficer 
must open the door to leave the OR. Hand hygiene with soap and water should be performed. N95 respirator should be removed. Hand hygiene should be performed. First surgical boots should be removed when leaving the transfer. Hand hygiene should be performed. In the dressing room, surgical cap and surgical uniform should be removed and placed in a special bag for ulterior decontamination, it is suggested take a bath before leaving the OR and underwear and shoes should be placed in a special bag for decontamination. Hand hygiene with alcohol-based solutions according to the WHO is recommended (Figure 2-3).

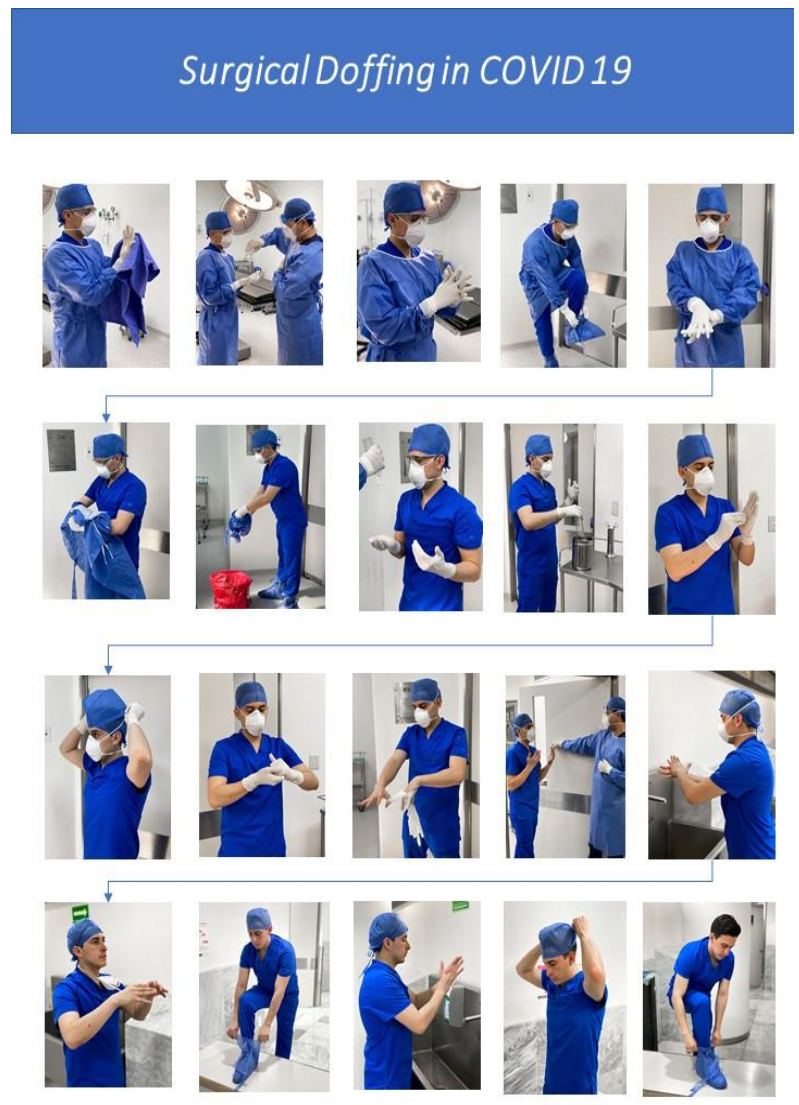

Figure 2: Surgical doffing in COVID-19.

\section{DISCUSSION}

Once the protocol was developed and tested using simulation in the operating room by the surgery department, it was implemented in the general surgery service with training consistent in master class with practice and graphic material provided to all members of the department in different sessions. The implementation started at the beginning of phase two of the pandemic in Mexico on 21 March 2020 to 5 May 2020, during this time 121 procedures were performed assuming all patients were possible COVID-19 infected and no member of the surgical team was infected and there were no cases of reoperation for an infected surgical wound. This protocol seeks to form a barrier to prevent the contact with aerosolization of the patient with the surgical team at the same time provide sterile environment for the safety of the patient. This amount of protective equipment can be difficult to wear to some people due to overheating in the surgery so it is recommended to practice before real procedures because removal or movement in contact with suspected or confirmed patient could end in infection. Limitations of the study lie in the rapid evolution of the pandemic and its evolving character, so it is necessary to carry out more research on surgical protection during surgeries suspected of COVID19, the pandemic brings to light new knowledge every day so these protocols must adapt according the new standards.

\section{How to Handrub?}

RUB HANDS FOR HAND HYGIENE! WASH HANDS WHEN VISIBLY SOILED (C) Duration of the entire procedure: $20-30$ seconds
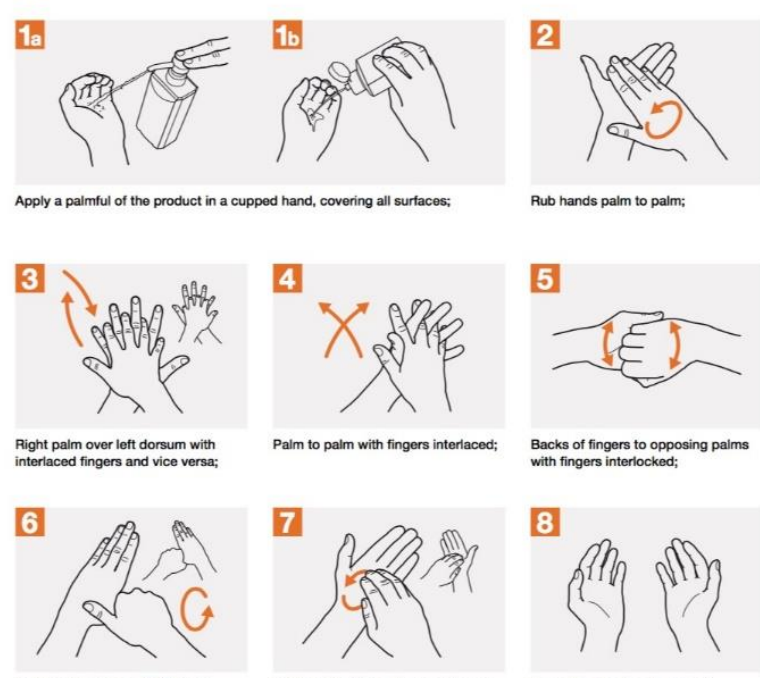
Rotational rubbing of left thumb
clasped in night palm and vice verse
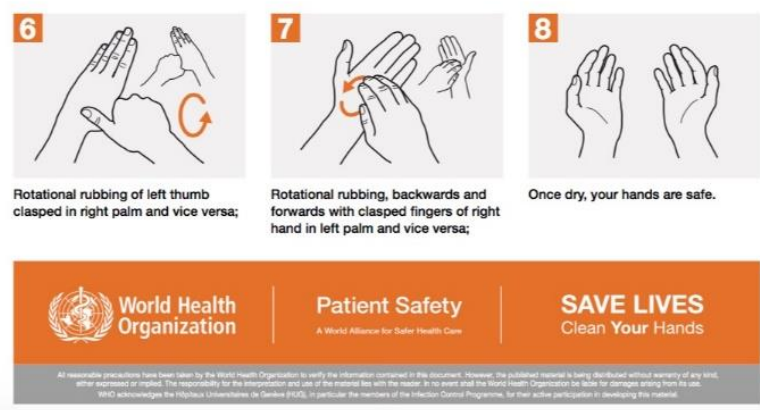

Figure 3: Hand rub instructions.

\section{CONCLUSION}

During this pandemic that has affected the world so much as health workers it is important to guarantee our safety, and as surgeons it is essential to preserve the aseptic principle in order to achieve a safe surgical procedure for patients and the surgical team. It is necessary to continue developing protocols to minimize risk of SARS-CoV-2 infection during surgery.

\section{Funding: No funding sources Conflict of interest: None declared Ethical approval: Not required}




\section{REFERENCES}

1. World Health Organisation (WHO) Coronavirus disease (COVID-19) outbreak webpage. Available at: https://www.who.int/emergencies/diseases/ novel-coronavirus-2019. Accessed on 18 April 2020.

2. Brat GA, Hersey S, Chhabra K, Gupta A, Scott J. Protecting Surgical Teams During the COVID-19 Outbreak: A Narrative Review and Clinical Considerations. Ann Surg. 2020;10:1097.

3. Surgical response in COVID-19. Available at: https://www.sages.org/recommendations-surgicalresponse-covid-19. Accessed on 18 April 2020.

4. Interim guidance for healthcare facilities: preparing for community transmission of COVID-19 in the United States. Available at: https://www.cdc.gov/ coronavirus/2019-ncov/healthcarefacilities/ guidance-hcf.html. Accessed on 10 April 2020.
5. COVID-19 PPE Guidance. Available at: https://repository.netecweb.org/exhibits/show/ppecons/item/697. Accessed on 18 April 2020.

6. ABS statement on training requirements during COVID-19. Available at: http://www.absurgery.org/ default.jsp?news_covid19_training. Accessed on 19 April 2020.

7. NIOSH fact sheet: the buddy system. Available at: https://www.cdc.gov/vhf/ebola/pdf/buddysystem.pdf. Accessed on 16 April 2020.

Cite this article as: Valmana OIG, Quero MMC, Loaeza EG, Sandoval JCL, Molina JAP, Ceballos MAR, et al. Protocol of sterile personal protective equipment for surgical personnel against SARS-

CoV-2 during the COVID-19 pandemic . Int J Res Med Sci 2020;8:4467-70. 